\title{
Hybrid Optical Beam-Former in Receiver Mode
}

\author{
Pham Q. Thai, Arokiaswami Alphones \\ Nanyang Technological University, Singapore City, Singapore \\ E-mail:ealphones@ntu.edu.sg \\ Received July 6, 2011; revised August 10, 2011; accepted August 22, 2011
}

\begin{abstract}
In this paper, an optical beam-former in receiving mode has been proposed and experimentally demonstrated. The requirement in system's hardware has been dramatically reduced using a hybrid approach between dispersive and non-dispersive delay. The proposed system is capable of supporting RF signals from L-band to $\mathrm{X}$-band, with large coverage and strong robustness against the grating's group delay ripples.
\end{abstract}

Keywords: Chirped Fiber Grating, Group Delay Ripple, Microwave Photonics, Optical Phased Array Antennas

\section{Introduction}

Despite having many advantages such as better directivity, higher gain, and beam-steering, traditional electrical controlled phase array antennas face serious problems working with large bandwidth RF signals at high frequencies. One of the most difficult obstacles to overcome is the "beam-squint" phenomenon. The beam direction of the array changes across the RF signal's bandwidth. For applications with large bandwidth and high frequency requirements, optical beam-forming is a promising candidate. Optical beam-formers inherit advantages characteristics such as large bandwidth, compactness, lightweight, and immunity to electromagnetic interference. More importantly, they can operate squint-free with wideband, high frequency signals, thus avoiding the most significant drawback of the traditional electrical beamformers.

Optical beamformers using chirped grating and optical delay lines are among the most prominent approaches for photonic beamforming [1]. However, in the former approach, the supported array size is limited since each element requires one tunable laser [2]. In the later approach, the length of the delay device results in many obstacles [3]. In order to combine the advantages of both approaches, optical beamforming systems using a combination of dispersive and non-dispersive delays have been proposed [4-7]. In [8], we have shown a hybrid approach between dispersive and non-dispersive delay for optical beam-former in transmitting mode. The novel system employing that method has dramatically reduced hardware requirements.
In this paper, the receiving schematic following our hybrid approach is presented. In our proposed receiver, the number of tunable laser is reduced by more than three times. The number of tunable optical delay line is reduced by two times. The required time delay for both devices is also greatly reduced. Experimental and simulation results have shown that the proposed beam-former can support signals with wide RF bandwidth from L-band to X-band. There were only slight distortions in the simulated radiation patterns in comparison with the ideal radiation patterns.

In [9], preliminary simulations and measurements of the proposed system were reported. In this article, many more details about the simulation radiation patterns and measurement results have been disclosed and discussed, especially about the distortions in radiation patterns. The system in this article has been improved from the previous system discussed in [10]. In [10], the combination of optical signals required intensive fine-tuning to ensure no interference between signals modulated at the same optical wavelength. In this paper, the problem has been overcome using polarization beam combiners.

Since the proposed system use chirped grating, the effect of group delay ripples (GDR) has also been addressed. As studied in [11,12], the GDR has a strong negative impact on the performance of optical beam-former using chirped grating in transmission operation. The study in [12] also suggested that grating may not be suitable for receiving operation. However, simulations have shown that our proposed hybrid system is more robust against GDR.

The paper is organized as described. The proposed 
beam-former is presented in Section 2. Experimental and simulation results of the proposed system are shown and discussed in Section 3. Finally, conclusions are drawn in Section 4.

\section{Principles}

In order to form the beam toward a particular direction $\theta$ in transmitting operation, an amount of time delay $t$ is required between two consecutive elements of a phased array antenna:

$$
t=d \sin (\theta) / c
$$

where $d$ is the spacing between elements and $c$ is the speed of light. Because of the additive or destructive combination of the transmitted signals from the elements, the main beam in far field would point at the desired direction.

In receiving operations, the above concept is also applied. It is assumed that the receiving signal approaches at the angle $\theta$. The delay between the received signals of two consecutive elements is $t$. By properly delaying the received signal at each element, the beam-former can pro- vide the strongest accumulated output signal. In a sense, delaying the received signal can be considered as pointing the main beam toward the desired direction.

The proposed beam-former in receiving mode is shown in Figure 1. The signal's delay at each element is also noted in that figure. The purpose of the beam-former is to correctly delay the signals, thus obtaining the strongest output signal after the photodiode.

The working principle has been discussed in [9]. However, for better clarity, a brief explanation is provided here. Firstly, the received signal from each element after the low noise amplifier is modulated with an optical signal through an electro-optic modulator. The first and third laser sources are tunable lasers, while the second and fourth laser sources are fixed wavelength lasers. The modulated optical signal for each modulator is noted in Figure 1.

Couplers are used to combine the modulated signals into several branches. Optical delay lines are used to delay the signal as shown in Figure 1. All optical delay lines are non-dispersive devices. The delay signals from the branches are then combined and passed through the multi-

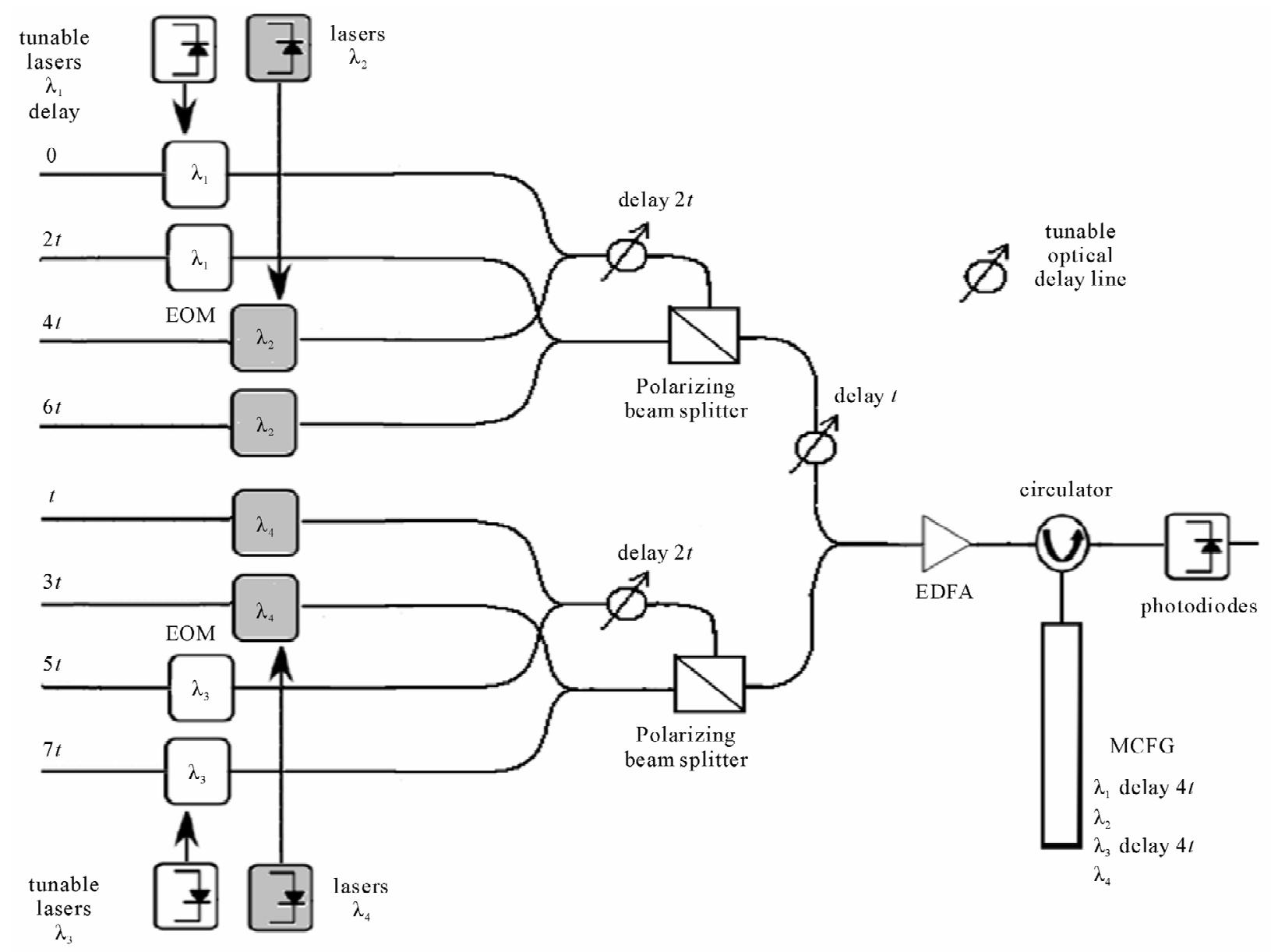

Figure 1. Hybrid optical beamformer in receiving mode. 
channel chirped fiber grating (MCFG). The reflectivity and group delay characteristic of the MCFG can be referred to in [11]. In a sense, the MCFG can be considered as a cascade of several nearly identical chirped gratings. Each optical wavelength falls into one channel of the MCFG. By tuning the wavelength, $\lambda_{1}$ is delayed by $4 t$ in comparison with $\lambda_{2}$, and $\lambda_{3}$ is delayed by $2 t$ in comparison with $\lambda_{4}$.

In order to avoid interferences, the optical signals with the same wavelength have to be of different polarizations. At the inputs of the polarizing beam splitter (PBS), the two input signals at the same wavelength are turned orthogonal via polarization controllers. The output signal after the PBS has two polarizations and can still be processed with the photo detector in the later stage.

As a result, all the input signals have same amount of $7 t$ delay in time. The accumulated output signal after the photodiode should be the strongest possible signal. In other words, by tuning the optical delay lines and the tunable lasers, the receiver has turned its main beam toward the signal's incoming direction.

Only four channels of the MCFG are used in this schematic. Since the MCFG has around 50 channels, it is possible to increase the supported array size by adding similar delay branches before the MCFG. In Table 1, the comparison between hardware's requirements of the hybrid system versus the typical systems is shown.

For optical beamformers using chirped grating, each element in the array requires one expensive tunable laser, which makes the system impractical. Optical beamformer using only optical delay lines is more cost-effective. However, the long delay requirement causes many disadvantages [13].

Our proposed approach, which utilizes a combination of both chirped grating and optical delay lines, has several advantages. The hybrid approach has been able to dramatically reduce the number of required tunable lasers and optical delay lines, as seen in Table 1. Moreover, the required delay for both the grating and delay line is greatly reduced. The proposed hybrid approach has been able to subdue the most prominent disadvantages of optical beamformers using chirped grating and optical delay line.
On the other hand, the scalability of our proposed system is limited. In receiving mode, combining modulated optical signals at the same wavelength may cause interferences. Polarization controller have been used to ensure that optical signal at the same wavelength have different polarization. As a result, each optical carrier signal can only be used two times. Therefore, although applying the same concept as in [8], the number of laser sources has to be increased in receiving operation.

\section{Experimental and Simulation Results}

In order to test the hybrid system, experiments have been conducted. Since there were not enough devices, improvising was made. Combining optical signal of different wavelengths does not distort the signal [14]. With proper polarizing, combining optical signal of the same wavelengths also does not cause distortion [15]. The MCFG helps prevent beatings [2]. On the other hand, the stability, precision, linearity, and the supported amount of time delay are among the most important parameters of a beamformer. Therefore, the set-up in Figure 2 was used to measure the delay capability in time domain of the proposed hybrid optical beamformer scheme. Each branch of the schematic in Figure 1 was measured at a time. For the branch with optical delay line, an optical delay was inserted between the modulator and the MCFG.

In the experiment, the wavelengths for four sources were $1547.503,1548.312,1549.112$ and $1549.917 \mathrm{~nm}$, respectively. The tuning step was $0.001 \mathrm{~nm}$. The optical signal occupied four separated channels of the MCFG. The grating was a MCFG from Teraxion, modeled as the Clear Spectrum DCX D061983.

The photodiode was use to convert the signal back into electrical domain. The output RF signals were then compared with the reference input RF signals. The tuning ranges of the tunable sources were from 1547.373 to $1547.643 \mathrm{~nm}$ for the first wavelength and from 1548.982 to $1549.252 \mathrm{~nm}$ for the third wavelength. The optical delay lines were tuned accordingly to the absolute delay between the wavelengths as explained in the previous section. The resulting delays between the RF output signals after the photodiodes at the outputs are shown in

Table 1. Comparison between systems' requirements in receiving mode.

\begin{tabular}{|c|c|c|c|c|}
\hline & $\begin{array}{l}\text { Number of optical } \\
\text { delay lines }\end{array}$ & $\begin{array}{l}\text { Maximum time delay } \\
\text { from delay line }\end{array}$ & $\begin{array}{l}\text { Number of } \\
\text { tunable lasers }\end{array}$ & $\begin{array}{l}\text { Maximum time delay } \\
\text { from chirped grating }\end{array}$ \\
\hline $\begin{array}{l}\text { Optical beamformer using chirped grating } \\
\qquad(4 / 8 / 16 \text { elements })\end{array}$ & $0 / 0 / 0$ & $0 / 0 / 0$ & $3 / 7 / 15$ & $3 t / 7 t / 15 t$ \\
\hline $\begin{array}{l}\text { Optical beamformer using optical delay line } \\
\qquad(4 / 8 / 16 \text { elements })\end{array}$ & $3 / 7 / 15$ & $3 t / 7 t / 15 t$ & $0 / 0 / 0$ & $0 / 0 / 0$ \\
\hline $\begin{array}{l}\text { Optical beamformer using the hybrid method } \\
\qquad(4 / 8 / 16 \text { elements })\end{array}$ & $1 / 3 / 7$ & $t / 2 t / 3 t$ & $1 / 2 / 4$ & $2 t / 4 t / 8 t$ \\
\hline
\end{tabular}




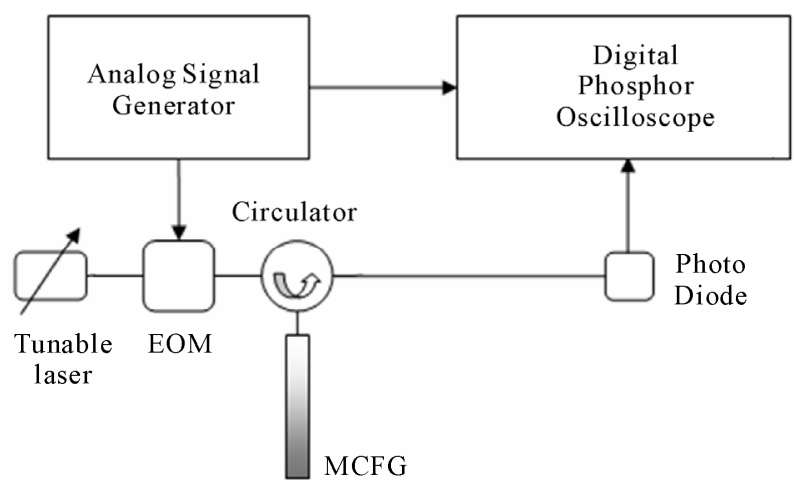

Figure 2. Experiment setup.

\section{Figures 3 and 4.}

As seen in those figures, the time delays are mostly linear. The results indicate that the tuning devices in the system, such as the laser sources and the optical lines, can be programmed to linearly and continuously tune.

The amount of delay stayed mostly unchanged at different RF signal frequencies, as shown in Figures 5 and 6. Considering the optical system as a black box, those results indicated that the system is able to support signals for L, S, C, and X-band arrays. The measured group delays of the two set of elements are also nearly the same since the MCFG channels have similar characteristics. The results have suggested that the hybrid optical beamformer system is flexible.

The measured data was imported into Matlab to simulate the radiation pattern. The simulated arrays were 8element arrays with half-wavelength spacing at $8 \mathrm{GHz}$ and $2 \mathrm{GHz}$. Using the measured data, the first array was able to steer from broadside to $58^{\circ}$, while the second array was able to steer from broadside to $17^{\circ}$. For the 8-element array, the MCFG is only needed to provide $4 t$ in time delay. A typical schematic using chirped grating requires the grating to provide $7 t$. As a result, given the same amount of delay from the MCFG, a typical beam-former using chirped grating can only steer the beam toward $29^{\circ}$ for $8 \mathrm{GHz} \mathrm{RF}$ signals and toward $10^{\circ}$ for $2 \mathrm{GHz} \mathrm{RF}$ signal, respectively.

In Figures 7 and 8, the simulated radiation pattern were compared with the ideal radiation patterns, where the there is no GDR and no amplitude fluctuations. The effect of mutual coupling was calculated in all cases. The time delay could be fine-tuned to correct the beam direction. There was less than $0.2^{\circ}$ and less than $0.5^{\circ}$ in main beam direction error for the $2 \mathrm{GHz}$ and $8 \mathrm{GHz}$ arrays, respectively. Both the beam width and the sidelobe level are affected by the signal's amplitude. In the system, each signal passing through one optical delay line suffered 1 $\mathrm{dB}$ loss. As a result, the main beam gain was decreased by $1.89 \mathrm{~dB}$, and the sidelobe level was increased by $0.3 \mathrm{~dB}$. In the worst case with additional amplitude fluctuations

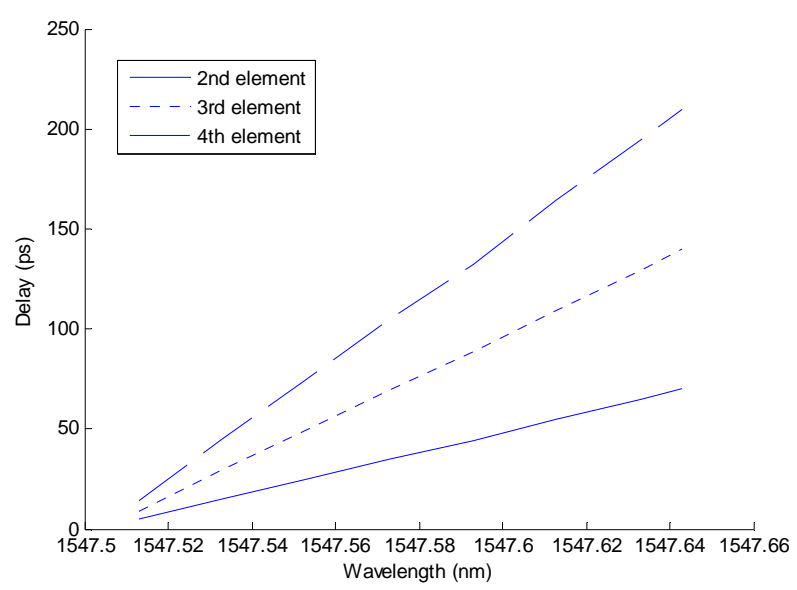

Figure 3. Time delays at the elements in the upper branch.

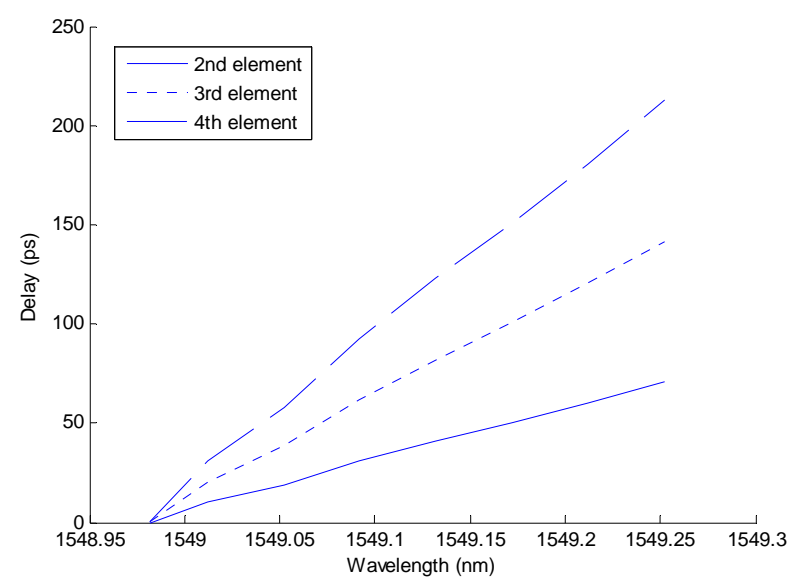

Figure 4. Time delays at the elements in the lower branch.

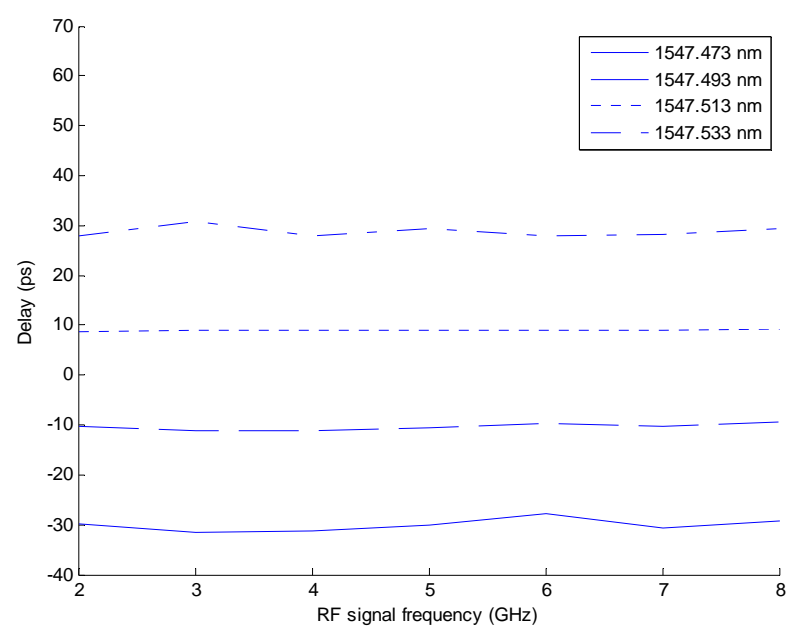

Figure 5. Time delay at different frequencies while tuning the first tunable laser.

from all devices, the main beam gain was decreased by $2.57 \mathrm{~dB}$ and the sidelobe level was increased by $0.6 \mathrm{~dB}$. The simulated pattern's $3 \mathrm{~dB}$ beam width is increased by 


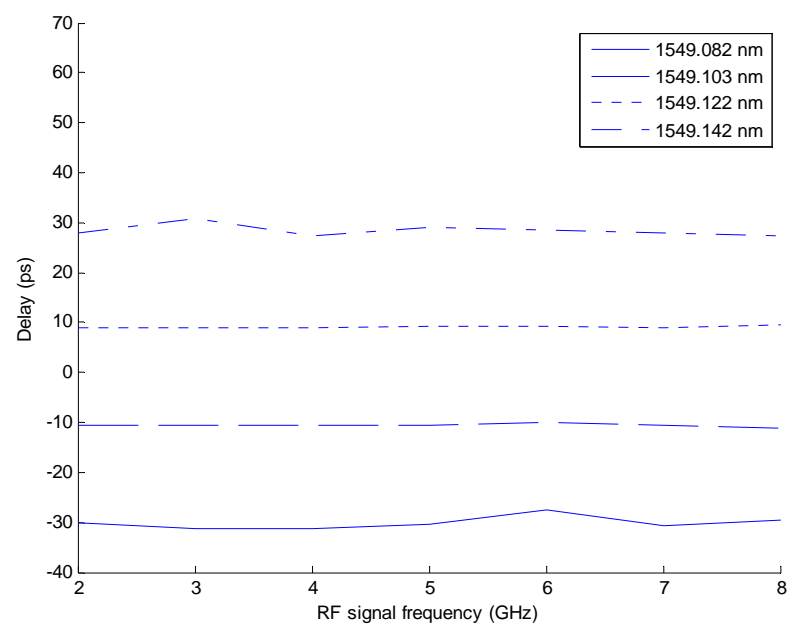

Figure 6. Time delay at different frequencies while tuning the second tunable laser.

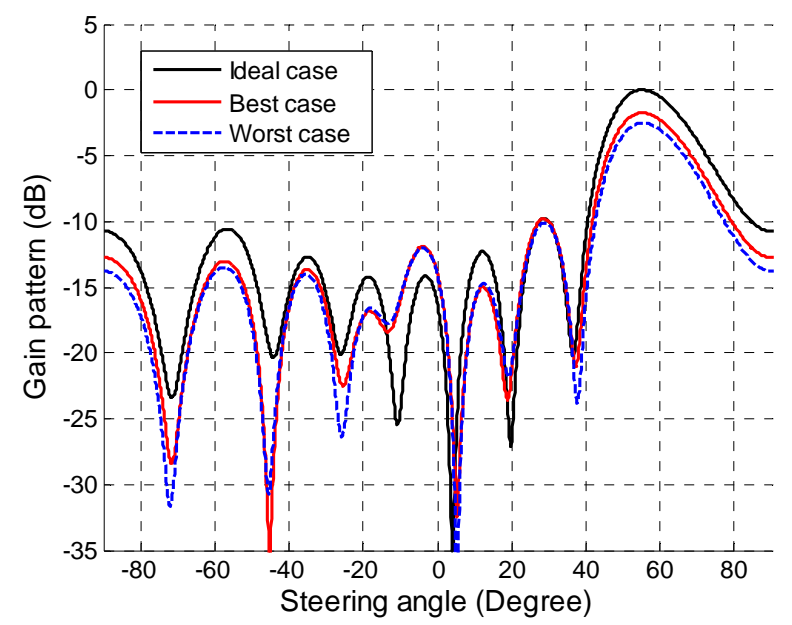

Figure 7. Comparison between the simulated and the ideal normalized radiation patterns for $8 \mathrm{GHz}$ signals at $55^{\circ}$.

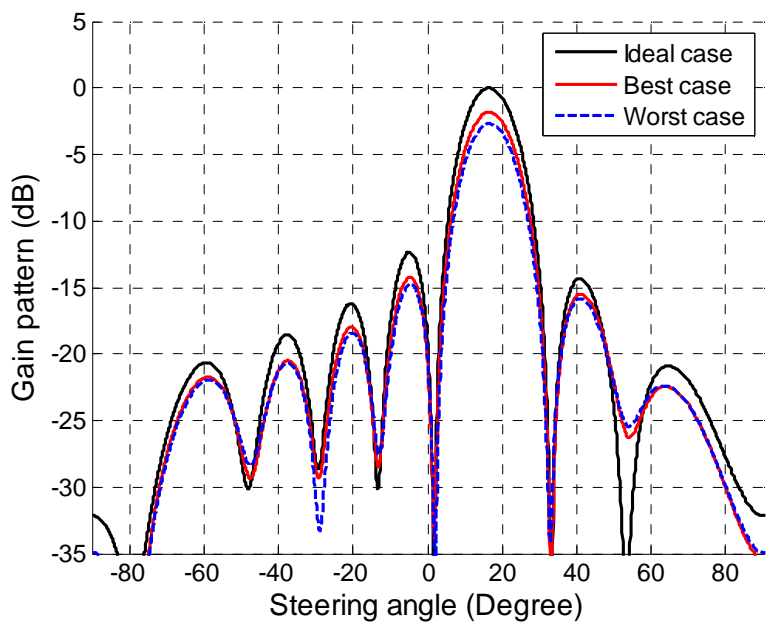

Figure 8. Comparison between the simulated and the ideal normalized radiation patterns for $2 \mathrm{GHz}$ signals at $17^{\circ}$. about $0.17^{\circ}$.

As analyzed in $[11,12]$, the fluctuations in group delay characteristic of the grating force many limitations on the optical beamformer using chirped grating. Since the proposed beamformer uses a chirped grating, its robustness against the GDR error should be tested. The main beam direction errors of the proposed system were compared with which of a typical optical beamformer using chirped grating.

In Figure 9, the errors in the hybrid system stay mostly the same for different array sizes. In comparison, the errors of the typical system quickly increase. The reason for that relates to the way the chirped grating is used. In a typical beamformer, each element is directly controlled via the delay of the grating, while the hybrid method only uses two channels of the grating to control the array. The less dependence on dispersive delay also reduces the negative effect of the group delay ripples.

In receiving, the quality and the signal's impulse response also have a higher priority. Previous report suggested that optical beamformers using chirped grating do not perform well in reception [12]. However, the system in [12] did not use the hybrid approach. The proposed system, on the other hand, is robust against GDR.

Using the same condition as in [12], linear frequency modulated (LFM) signals are considered. The details about LFM can be found in [16]. In short, this particular type of signal is highly recommended for its resolution and detection range. The radar systems using those signals place a strong emphasis on the quality of received signal's impulse response $[17,18]$. The change in the peak's position of the impulse response may cause false distance's estimation, while the rise in sidelobe level may conceal targets.

The fluctuation in group delay causes symmetrical re-

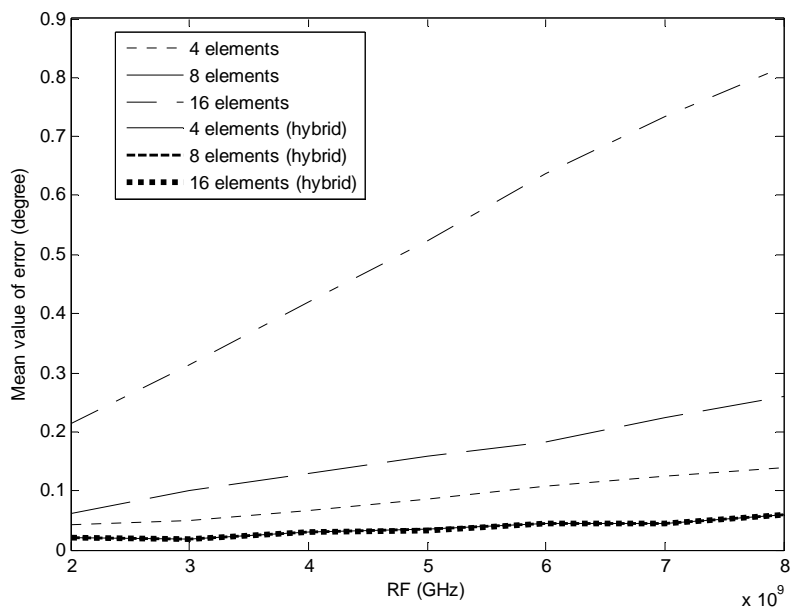

Figure 9. Main beam direction error at different RF signal frequencies and different array sizes. 
sponses of the received signal around the non-error signal. This phenomenon, called "paired echo", is caused by dispersion error as studied in [18]. Depending on the disturbance's period, the $3 \mathrm{~dB}$ width of the main lobe may increase or new sidelobes may arise in the impulse response.

Preliminary study was done in [9]. Further analysis has shown that the negative effect of GDR is nearly nonexistent. In the hybrid system, only two wavelengths were tuned across a small region in the grating. Furthermore, only a part of the total delay was from the grating. As a result, the influence from GDR was minimized. Although the echo was presented at several different positions in time domain, the displacement of them was greatly reduced to a few picoseconds, while the LFM pulse duration is usually in microseconds. Since the scaling is so distinctive, the negative effects such as distortions from additive and subtractive combinations are negligible. The study in [12] also agreed that small ripples do not reduce the system performance. However, the system in [12] could not achieve this goal since every element was under the influence from GDR.

For example, a simple case of LFM has been considered. In Figure 10, the impulse response of a LFM with bandwidth of $1 \mathrm{GHz}$ and pulse duration of $10 \mu$ s with and without GDR are shown. The centre frequency was chosen as $7.5 \mathrm{GHz}$. As seen in the figure, the two cases were almost undistinguishable. All the important factors such as the $3 \mathrm{~dB}$ width of the main lobe, the main lobe position, the number of sidelobes, and the sidelobe level are mostly unchanged. The change in main lobe level across the signal bandwidth was only around $0.015 \mathrm{~dB}$.

\section{Conclusions}

In this paper, an optical beam-former in receiving mode

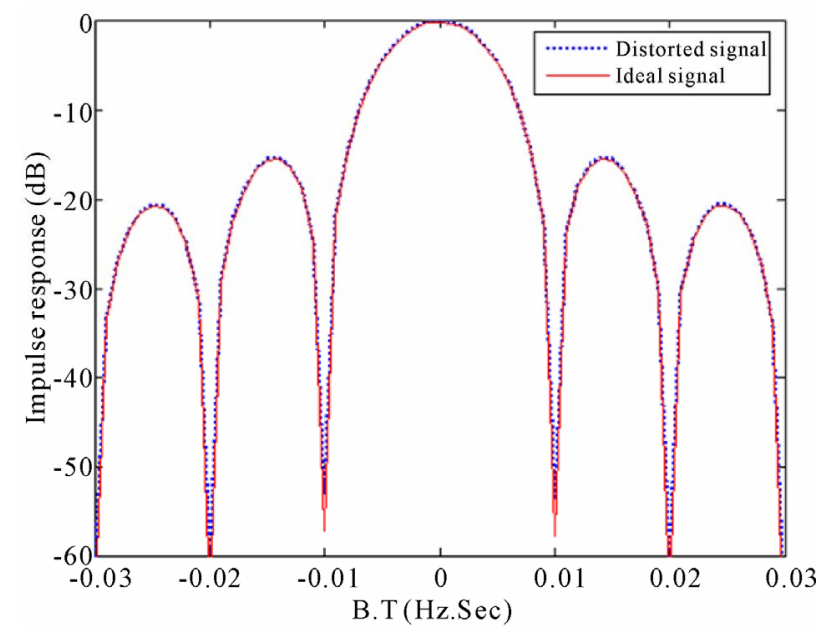

Figure 10. Impulse response of the LFM signal under the influence of GDR. using a hybrid approach between dispersive and nondispersive delay has been introduced. The support RF signal covers a huge bandwidth from L-band to X-band. In addition, the proposed beam-former has provided such promising capabilities using incredibly less requirement in optical hardware and time delay. Analysis about the effect of GDR has also indicated that the proposed system is more robust against GDR.

\section{References}

[1] N. A. Riza, Selected Papers on Photonic Control Systems for Phased Array Antennas, 694 SPIE Optical Engineering Press, 1997.

[2] D. B. Hunter, M. E. Parker and J. L. Dexter, "Demonstration of a Continuously Variable True-Time Delay Beamformer Using a Multichannel Chirped Fiber Grating," IEEE Transactions on Microwave Theory and Techniques, Vol. 54, No. 2, 2006, pp. 861-867. doi:10.1109/TMTT.2005.863056

[3] M. Y. Frankel, P. J. Matthews and R. D. Esman, "TwoDimensional Fiber-Optic Control of a True Time-Steered Array Transmitter," IEEE Transactions on Microwave Theory and Techniques, Vol. 44, No. 12, 1996, pp. 2696 2702. doi: $10.1109 / 22.554632$

[4] N. A. Riza and N. Madamopoulos, "Photonic Time Delay Beamforming Architectures Using Polarization Switching Arrays," In: D. R. Pape, Ed., Advances in Optical Information Processing VII, Proceedings of the Society of Photo-Optical Instrumentation Engineers (Spie), SPIEInternational Society Optical Engineering, Bellingham, 1996, pp. 186-197.

[5] N. A. Riza and N. Madamopoulos, "Phased-Array Antenna, Maximum-Compression, Reversible Photonic Beam Former with Ternary Designs and Multiple Wavelengths," Applied Optics, Vol. 36, No. 5, 1997, pp. 983996. doi:10.1364/AO.36.000983

[6] N. A. Riza, M. A. Arain and S. A. Khan, "Hybrid Analog-Digital Variable Fiber-Optic Delay Line," Journal of Lightwave Technology, Vol. 22, No. 2, 2004, pp. 619624. doi:10.1109/JLT.2004.824383

[7] B. M. Jung and H. P. Yao, “A Two-Dimensional Optical True Time-Delay Beamformer Consisting of a Fiber Bragg Grating Prism and Switch-Based Fiber-Optic Delay Lines," IEEE Photonics Technology Letters, Vol. 21, No. 9-12, 2009, pp. 627-629. doi:10.1109/LPT.2009.2015275

[8] P. Q. Thai, A. Alphones and D. R. Lim, "A Novel Simplified Dual Beam-Former Using Multichannel Chirped Fiber Grating and Tunable Optical Delay Lines," Journal of Lightwave Technology, Vol. 26, No. 13-16, 2008, pp. 2629-2634. doi:10.1109/JLT.2008.927152

[9] P. Q. Thai and A. Alphones, "Hybrid Optical Beamformers and Their Robustness Against Group Delay Ripples Errors," IEEE Topical Meeting on Microwave Photonics (MWP), Montreal, 5-9 October 2010, pp. 276-279.

[10] A. Alphones and T. P. Quang, "Hybrid Approach for 
Optical Beamforming for Phased Array," Microwave Conference Proceedings (APMC), Asia-Pacific 2010, Singapore City, 7-10 December 2010, pp. 311-317.

[11] P. Q. Thai, A. Alphones and D. R. Lim, "Limitations by Group Delay Ripple on Optical Beam-Forming With Chirped Fiber Grating," Journal of Lightwave Technology, Vol. 27, No. 24, 2009, pp. 5619-5625. doi:10.1109/JLT.2009.2032786

[12] R. Rotman, O. Raz and M. Tur, "Requirements for True Time Delay Imaging Systems with Photonic Components," IEEE International Symposium on Phased Array Systems and Technology 2003, New York, 14-17 October 2003, pp. 193-198.

[13] R. Soref, "Optical Dispersion Technique for Time-Delay Beam Steering," Applied Optics, Vol. 31, No. 35, 1992, pp. 7395-7397. doi:10.1364/AO.31.007395

[14] J. Capmany, B. Ortega and D. Pastor, "A Tutorial on Microwave Photonic Filters," Journal of Lightwave Technology, Vol. 24, No. 1, 2006, pp. 201-229.

\section{doi:10.1109/JLT.2005.860478}

[15] J. Q. Zhou, S. Aditya, P. Shum, L. Xia and B. P. Parhusip, "Wide-Range Continuously Tunable Microwave PhoTonic Filter Using High-Birefringence Linearly Chirped Fiber Bragg Grating and Polarization Beamsplitters," Optical Engineering, Vol. 48, No. 1, 2009, Article ID: 010502 .

[16] M. Skolnik, "Radar Handbook," 3rd Edition, McGrawHill Professional, Boston, 2008.

[17] A. Kawalec, C. Lesnik, W. Komomiczak, W. Czamecki and J. Pietrasiniski, "Influence of the Displacement Effect on Compressed LFM Signal Parameters," In: C. A. Brebbia and G. M. Carlomagno, Eds., Computational Methods and Experimental Measurements XIII, Wit Transactions on Modelling and Simulation, 2007, pp. 769-778.

[18] S. Darlington, W. J. Albersheim and A. C. Price, "The Theory and Design of Chirp Radars," The Bell System Technical Journal, Vol. 39, No. 4, 1960, pp. 745-802. 